\title{
Relação dos saltos vertical, horizontal e sêxtuplo com a agilidade e velocidade em crianças
}

CDD. 20.ed. 152.3

796.426
Diogo Henrique Constantino COLEDAM" Gustavo Aires de ARRUDA* Júlio Wilson dos-SANTOS ${ }^{* *}$ Arli Ramos de OLIVEIRA*
*Universidade Estadual de Londrina.

**Departamento de Educação Física, Universidade Estadual Paulista - Bauru.

\section{Resumo}

Os objetivos do presente estudo foram: 1) verificar a relação dos saltos vertical, horizontal e sêxtuplo com a agilidade e velocidade de 5,10 e $25 \mathrm{~m} ; 2$ ) verificar a capacidade desses saltos em predizer o desempenho da agilidade e velocidade de 5, 10 e $25 \mathrm{~m}$ em crianças. Vinte e oito meninos $(9,47 \pm 0,64$ anos) e 30 meninas $(9,69 \pm 0,70$ anos) foram avaliados. Os valores de correlação entre a agilidade, velocidade de 5,10 e $25 \mathrm{~m}$ foram, respectivamente, $r=0,63,0,51,0,44$ e 0,64 com o salto vertical, $r=0,68,0,62$, 0,28 e 0,62 com o salto sêxtuplo, e $r=0,60,0,50,0,26$ e 0,57 com o salto horizontal. 0 salto vertical e o salto sêxtuplo foram capazes de predizer o desempenho da agilidade e da velocidade de $25 \mathrm{~m}(\mathrm{p}<$ $0,05)$. Além disso, demonstraram capacidade de predizer a velocidade de 5 e $10 \mathrm{~m}$, respectivamente $(p<$ $0,05)$. Os testes de salto vertical e sêxtuplo podem ser utilizados para avaliação e controle do treinamento com crianças praticantes de atividades que demandam agilidade e velocidade, uma vez que ambos os saltos predisseram o desempenho da agilidade e velocidade, o que não ocorreu com o salto horizontal.

Palavras-chave: Corrida; Desempenho motor; Potência de membros inferiores.

\section{Introdução}

A capacidade motora força é estudada em crianças devido a sua importância tanto para a saúde como para o desempenho atlético ${ }^{1}$. A força muscular é definida como a alteração ou tendência de alteração do estado de repouso ou de movimento de uma situação resultante da ação muscular².

Considerando as variáveis que influenciam a produção de força, tais como a resistência ou massa a ser vencida, aceleração e velocidade do movimento, a força pode ser manifestada de maneiras diferentes. Força muscular máxima é o valor mais elevado de força que o sistema neuromuscular é capaz de produzir, alcançada em aproximadamente $0,4 \mathrm{~s}^{3}$. No entanto, em muitos movimentos esportivos não há tempo suficiente para atingir a força muscular máxima. Nesse contexto aplica-se a força explosiva, entendida como a relação entre a maior expressão de força atingida em um determinado tempo disponível e o tempo necessário para alcançá-la ${ }^{3}$. A potência muscular representa a razão entre um determinado trabalho realizado por segundo e pode ser avaliada em um único ou uma série de movimentos corporais ${ }^{4}$. Um mecanismo fisiológico que contribui para a produção de força explosiva é o ciclo de alongamento e encurtamento, responsável por gerar energia elástica a partir da contração muscular excêntrica seguida por uma contração concêntrica ${ }^{5}$.

Devido às diversas formas que pode ser manifestada, a força tem sido estudada em crianças por meio de diferentes métodos de avaliação, dentre eles a execução de saltos, levantamento de pesos, dinamômetro hidráulico e isocinético ${ }^{6}$. Os saltos vertical, horizontal e sêxtuplo têm sido utilizados como indicadores da força dos membros inferiores em crianças e adolescentes, uma vez que demonstraram ser sensíveis ao treinamento de força $^{7-9} \mathrm{e}$ ao treinamento específico de modalidade esportiva handebol ${ }^{10}$. Além de apresentarem sensibilidade a diferentes tipos de treinamento, os testes envolvendo saltos são utilizados por serem específicos às ações de diferentes modalidades esportivas ${ }^{11}$. 
Assim como a força, a agilidade e a velocidade são capacidades motoras importantes para o desempenho atlético de diversas modalidades esportivas. Além disso, o desempenho da agilidade e da velocidade depende da produção de força máxima, da potência e da energia elástica produzida pelo ciclo de alongamento e encurtamento ${ }^{12-13}$, motivo pelo qual a relação entre a força muscular, agilidade e velocidade têm sido investigadas. Os testes de salto vertical e horizontal apresentam correlação linear de fraca a moderada com a agilidade, em atletas homens da modalidade voleibol e futebol ${ }^{14-15}$ e mulheres atletas de futebol adultas e adolescentes ${ }^{11}$. Para a velocidade e o salto vertical, essa correlação pode variar de fraca a muito forte em atletas de rúgbi homens ${ }^{16} \mathrm{e}$ atletas de ambos os sexos de diferentes modalidades esportivas ${ }^{12}$. Entre a velocidade e o salto horizontal, os valores de correlação variam de fraca a forte em homens atletas de futebol ${ }^{15} \mathrm{e}$ adolescentes atletas de varias modalidades esportivas ${ }^{17}$.

Apesar de alguns estudos já terem descrito as relações entre o salto vertical e horizontal com a agilidade e a velocidade, a inconsistência dos resultados obtidos

\section{Método}

\section{Amostra}

Participaram voluntariamente do estudo 58 crianças (28 meninos e 30 meninas), regularmente matriculadas no $5^{\circ}$ ano da rede municipal de ensino da cidade de Nova Europa - SP. A amostra foi escolhida de forma intencional, de acordo com a disponibilidade de acesso e os critérios de inclusão no estudo foram: não possuir nenhuma limitação física/ortopédica que impedisse a realização dos testes físicos e ter frequência mínima de 85\% nos dois últimos meses nas aulas de Educação Física. Os pais ou responsáveis pelos participantes assinaram um Termo de Consentimento Livre e Esclarecido de acordo com a Resolução no 196/96 do Ministério da Saúde especificando todos os procedimentos do estudo. O projeto de pesquisa foi aprovado pelo Comitê de Ética em Pesquisa da Universidade Estadual Paulista UNESP, protocolo no 11017/46/01/10.

\section{Procedimentos}

Todos os procedimentos do estudo foram realizados na escola onde os participantes estavam matriculados. Foram realizadas medidas de massa corporal, por meio de uma balança digital com precisão de $100 \mathrm{~g}$, e a por estudos prévios sugerem que estas relações ainda necessitam ser investigadas. Além disso, a maioria dos estudos sobre a relação entre esses parâmetros de desempenho foi realizada com adultos de diferentes modalidades esportivas ${ }^{18-19}$ e a capacidade dos testes de salto vertical, horizontal e sêxtuplo em predizer o desempenho da agilidade e da velocidade de 5, 10 e 25 $m$ ainda não foi descrita em crianças. As informações acerca do tipo de salto que melhor prediz o desempenho da agilidade e velocidade irão auxiliar na escolha do teste de força que seja mais específico para avaliar e controlar o treinamento de crianças envolvidas em modalidades em que a agilidade e velocidade são determinantes. Além disso, identificar a magnitude da contribuição dos saltos para o desempenho da velocidade e agilidade torna possível prescrever o treinamento de força de forma específica para aumento dessas capacidades ${ }^{20}$.

Desse modo, os objetivos do presente estudo foram: 1 ) verificar a relação dos saltos vertical, horizontal e sêxtuplo com a agilidade e velocidade de 5, 10 e $25 \mathrm{~m} \mathrm{e}$; 2) verificar a capacidade desses saltos em predizer o desempenho da agilidade e velocidade de 5, 10 e $25 \mathrm{~m}$ em crianças.

estatura foi verificada com um estadiômetro portátil fixado em uma parede, com precisão de $0,1 \mathrm{~cm}$.

Os testes de salto vertical, horizontal e sêxtuplo, agilidade e corrida de 5, 10 e $25 \mathrm{~m}$ foram realizados em uma quadra poliesportiva que possuía piso antiderrapante. Os participantes usaram roupas e calçados adequados à prática de exercícios físicos. Para o teste de salto vertical foi utilizada uma parede lisa de $3 \mathrm{~m}$ de altura. Para medir a distância dos saltos vertical, horizontal, sêxtuplo e a distância das corridas de 5, 10 e $25 \mathrm{~m}$ foi utilizada uma trena de $30 \mathrm{~m}$ (Profield $^{\circledR}$, São Paulo, Brasil). Os tempos nos testes de agilidade e corrida de 5, 10 e $25 \mathrm{~m}$ foram registrados por meio de um cronômetro manual com precisão de décimos de segundo (Casio ${ }^{\circledR}$, HS-7, Japão).

Anteriormente à coleta de dados, todos os participantes receberam informações sobre os procedimentos dos testes, através de demonstraçôes feitas por um dos pesquisadores, e foram submetidos a três tentativas em cada teste, em dois dias diferentes, para controlar os efeitos de aprendizagem. Durante a coleta de dados, cada teste foi realizado em um dia, com intervalo de $24 \mathrm{~h}$ entre cada avaliação, e a cada dois dias houve um intervalo de $48 \mathrm{~h}$ ou 72 h, conforme descrito no QUADRO 1. 
QUADRO 1 - Cronograma dos dias de realização dos testes de Salto Vertical (SV), Salto Horizontal (SH), Salto Sêxtuplo (SS), agilidade (AG), velocidade de 5 (V5), 10 (V10) e 25 m (V25).

\begin{tabular}{|c|c|c|c|c|c|c|c|c|c|c|c|}
\hline Dia & 1 & 2 & 3 & 4 & 5 & 6 & 7 & 8 & 9 & 10 & 11 \\
\hline Teste & SV & SH & - & SS & AG & - & - & V-5 & V-10 & - & V-25 \\
\hline
\end{tabular}

No dia de realização dos testes os participantes foram orientados a não realizar nenhum esforço físico vigoroso. A coleta de dados foi realizada pelo mesmo pesquisador, com experiência prévia na aplicação de testes motores, e a melhor das três tentativas foi considerada para análise. Anteriormente ao início de cada teste, os participantes realizaram um aquecimento com duração de $10 \mathrm{~min}$, que compreendeu 4 min de corrida de intensidade leve e duas séries $(20 \mathrm{~s})$ de seis exercícios dinâmicos (elevação de joelho, elevação de tornozelo atrás, "skipping", chute baixo para frente, chute baixo para trás e deslocamento lateral).

\section{Descrição dos testes}

Salto vertical: Para a realização do teste de salto vertical (SV) foi fixada uma fita métrica de três metros em uma parede lisa. $\mathrm{O}$ avaliado posicionava-se lateralmente à superfície graduada, com as plantas dos pés totalmente apoiadas sobre o solo, e com um braço completamente estendido acima da cabeça, onde foi marcado o ponto mais alto alcançado com o dedo médio. Para facilitar a marcação, foi utilizado pó de giz na extremidade dos dedos. A partir da posição ortostática, a execução consistiu em flexionar os joelhos e executar o salto contra movimento, com auxílio dos braços, e tocar o ponto mais alto possível na parede. A marcação foi feita com precisão de 0,5 $\mathrm{cm}$. O valor foi calculado pela diferença da maior altura alcançada (com salto) e a altura parado, com os valores expressos em centímetros.

Salto horizontal: Anteriormente à execução do salto, uma fita métrica foi fixada ao solo, paralelamente ao local onde o avaliado iria realizar o salto. Primeiramente, o avaliado se manteve parado, com os pés paralelos e afastados na mesma distância que a largura dos quadris. A parte anterior dos pés foi posicionada na linha de partida. $\mathrm{O}$ teste de salto horizontal $(\mathrm{SH})$ também foi executado com o salto contra movimento, com auxílio dos braços, seguido de um impulso simultâneo a partir da flexão dos joelhos, para atingir o ponto mais distante possível. A medida foi feita entre o ponto de partida e o calcanhar do pé mais próximo do ponto inicial, com precisão de $0,5 \mathrm{~cm}$.
Salto sêxtuplo: $\mathrm{O}$ avaliado iniciou o teste na posição em pé, parado atrás da linha inicial demarcada no solo, com as pernas na posição antero-posterior. $\mathrm{O}$ início do pé posicionado à frente se manteve no início da linha inicial. $\mathrm{O}$ avaliado realizou um salto contra movimento com auxílio dos braços, seguido de outros seis saltos unilaterais de forma alternada (direita / esquerda) e finalizou o teste no sexto salto com os dois pés. A medida foi realizada entre o ponto de partida e o calcanhar do pé mais próximo do ponto inicial, com precisão de $0,5 \mathrm{~cm}$.

Agilidade: A avaliação da agilidade (AG) foi feita por meio do teste de Vai-e-vem ("Shuttle run"). O teste consiste em percorrer a distância de 9,14 m duas vezes, indo e voltando (4 x 9,14 m). Atrás de uma das linhas divisórias do percurso foram colocados dois blocos de madeira. O objetivo do teste é buscar os dois blocos de madeira, um de cada vez, com uma das mãos, e colocá-los após a linha inicial, no menor tempo possível. $\mathrm{O}$ cronômetro foi acionado após o indivíduo ultrapassar a linha de partida e foi finalizado quando o segundo aparato de madeira foi deixado após a linha de partida.

Velocidade de 5, 10 e 25 m: Para verificar a velocidade foram utilizadas as corridas de velocidade máximas de 5 (V5), 10 (V10) e 25 m (V25). Um estímulo visual foi utilizado para a partida e a cronometragem foi iniciada com o movimento de partida do indivíduo, a partir da posição parada, com as pernas na posição antero-posterior. $\mathrm{O}$ avaliador ficou posicionado no final de cada percurso dos testes V5, V10 e V25, para a tomada do tempo da corrida.

\section{Reprodutibilidade de testes}

A reprodutibilidade dos testes de salto vertical, salto horizontal, salto sêxtuplo, agilidade e velocidade de 5, 10 e 25 m foi testada em 15 participantes. Os mesmos procedimentos de aprendizagem descritos foram realizados e os participantes realizaram três tentativas de cada teste, em dois dias, com um intervalo de $48 \mathrm{~h}$. A reprodutibilidade de cada teste está descrita na TABELA 1 . Todos os testes apresentaram alta reprodutibilidade, com coeficientes de correlação Intraclasse variando de 0,84 para a velocidade de $5 \mathrm{~m}$ a 0,96 para o salto vertical. 
TABELA 1 - Reprodutibilidade dos testes utilizados no estudo.

\begin{tabular}{lllc}
\cline { 2 - 4 } $\mathrm{CCI}=$ Coeficiente de & Teste & CCI & IC95\% \\
\cline { 2 - 4 } Correlação Intraclasse; & Salto vertical & 0,96 & $0,91-0,99$ \\
IC95\% Intervalo de & Salto horizontal & 0,90 & $0,71-0,96$ \\
confiança de 95\% do & Salto sêxtuplo & 0,91 & $0,84-0,98$ \\
coeficiente de correlação & Agilidade & 0,93 & $0,80-0,97$ \\
Intraclasse. & Velocidade 5 m & 0,84 & $0,61-0,96$ \\
& Velocidade 10 m & 0,95 & $0,85-0,98$ \\
& Velocidade 25 m & 0,95 & $0,87-0,98$ \\
\hline
\end{tabular}

\section{Análise estatística}

O cálculo do tamanho amostral foi realizado de acordo com o $\mathrm{r}^{2}$ de 0,40 para a regressão múltipla, alpha $=0,05$, beta $=20 \%$ e número de variáveis preditoras $=3$, utilizando o "software" G*Power 3.0. Os pressupostos de linearidade, normalidade, homocedasticidade e ausência de autocorrelação dos resíduos e multicolinearidade das variáveis independentes (todas as variáveis incluídas no modelo apresentaram VIF < 1,5) foram aceitos. A análise descritiva foi realizada por meio da média e desvio padrão. Para comparar as variáveis entre os meninos e as meninas foi utilizado o Teste t de Student para amostras independentes. A relação entre as variáveis foi verificada por meio da Correlação linear de Pearson (r) e os seus respectivos Intervalos de Confiança de 95\% foram apresentados. A interpretação foi realizada conforme TRITSCHLER ${ }^{21}$, onde: $<0,30$ = pouca ou nenhuma correlação; 0,30 a 0,49 = fraca; 0,50 a $0,69=$ moderada; 0,70 a 0,89: forte; $>0,90$ = muito forte, valores positivos e negativos são interpretados da mesma forma. Para verificar a capacidade das variáveis independentes (salto vertical, horizontal e sêxtuplo) em predizer as variáveis dependentes (agilidade, velocidade de 5, 10 e 25 m) foi realizada a Regressão Linear Múltipla pelo método Stepwise para cada variável independente. Para analisar a reprodutibilidade dos testes foi utilizado o Coeficiente de Correlação Intraclasse com efeito aleatório de um fator. Em todos os casos a significância adotada foi de $5 \%$.

\section{Resultados}

Na TABELA 2 estão apresentados os valores de idade, massa corporal, estatura, salto vertical, horizontal, sêxtuplo, agilidade, velocidade de 5, 10 e $25 \mathrm{~m}$ para meninos e meninas. Foram verificadas

diferenças significativas para a agilidade e velocidade de $25 \mathrm{~m}$, sendo que os meninos apresentaram maior desempenho para a agilidade e as meninas para a velocidade de $25 \mathrm{~m}$.

TABELA 2 - Características dos sujeitos do estudo em valores de média e desvio padrão.

${ }^{*} \mathrm{p}<0,05$ vs meninas.

\begin{tabular}{lccc}
\hline & Meninos $(\mathbf{n}=\mathbf{2 8})$ & Meninas $(\mathbf{n}=\mathbf{3 0})$ & Total $(\mathbf{n}=\mathbf{5 8})$ \\
\hline Idade (anos) & $9,47(0,64)$ & $9,69(070)$ & $9,58(0,67)$ \\
Massa corporal $(\mathrm{kg})$ & $31,29(6,88)$ & $33,26(6,80)$ & $29,45(5,94)$ \\
Estatura $(\mathrm{m})$ & $1,37(0,11)$ & $1,36(0,15)$ & $1,36(0,13)$ \\
Índice de massa corporal $\left(\mathrm{kg} / \mathrm{m}^{2}\right)$ & $17,33(4,33)$ & $18,05(2,78)$ & $17,67(3,66)$ \\
Salto Vertical $(\mathrm{cm})$ & $26,66(5,26)$ & $25,55(6,14)$ & $26,10(5,70)$ \\
Salto Horizontal $(\mathrm{cm})$ & $161,57(15,14)$ & $155,44(21,39)$ & $158,51(18,27)$ \\
Salto Sêxtuplo $(\mathrm{cm})$ & $871,14(108,89)$ & $829,29(120,72)$ & $850,22(114,80)$ \\
Agilidade $(\mathrm{s})$ & $11,23(0,73)^{*}$ & $11,67(0,88)$ & $11,45(0,80)$ \\
Velocidade de $5 \mathrm{~m}(\mathrm{~m} / \mathrm{s})$ & $3,52(0,22)$ & $3,39(0,27)$ & $3,45(0,25)$ \\
Velocidade de $10 \mathrm{~m}(\mathrm{~m} / \mathrm{s})$ & $4,09(0,55)$ & $4,00(0,36)$ & $4,05(0,46)$ \\
Velocidade de $25 \mathrm{~m}(\mathrm{~m} / \mathrm{s})$ & $4,84(0,31) *$ & $4,57(0,36)$ & $4,70(0,34)$ \\
& & &
\end{tabular}

46 • Rev Bras Educ Fís Esporte, (São Paulo) 2013 Jan-Mar;27(1):43-53 
$\mathrm{Na}$ TABELA 3 são apresentados os valores de correlação entre salto vertical, horizontal, sêxtuplo, agilidade, velocidade de 5, 10 e $25 \mathrm{~m}$. O salto vertical apresentou valores de correlação variando de $\mathrm{r}=0,44$ para a velocidade de $10 \mathrm{~m}$ a r $=0,64$ para a velocidade de $25 \mathrm{~m}$ e o salto sêxtuplo apresentou valores que variaram de $\mathrm{r}=0,28$ para a velocidade de $10 \mathrm{~m}$ a $\mathrm{r}=$ 0,68 para a agilidade. Com relação ao salto horizontal, o menor valor foi de $r=0,26$ para a velocidade de 10 $\mathrm{m}$ e o maior de $\mathrm{r}=-0,60$ para a agilidade.

TABELA 3 - Coeficientes de correlação e intervalo de confiança 95\% entre as variáveis: Salto Vertical (SV), Salto Horizontal (SH), Salto Sêxtuplo (SS), agilidade (AG), velocidade de 5 (V5), 10 (V10) e 25 m (V25).

\begin{tabular}{|c|c|c|c|c|c|c|}
\hline & SS & SH & $\mathrm{AG}$ & V5 & V10 & V25 \\
\hline SV & $\begin{array}{c}0,671^{* *} \\
(0,50-0,79)\end{array}$ & $\begin{array}{c}0,734^{* *} \\
(0,59-0,83)\end{array}$ & $\begin{array}{c}-0,632^{* *} \\
(-0,76-0,44)\end{array}$ & $\begin{array}{c}0,507^{* *} \\
(0,29-0,68)\end{array}$ & $\begin{array}{c}0,435^{* *} \\
(0,20-0,62)\end{array}$ & $\begin{array}{c}0,639^{* *} \\
(0,46-0,77)\end{array}$ \\
\hline SS & - & $\begin{array}{c}0,767^{* *} \\
(0,64-0,86)\end{array}$ & $\begin{array}{c}-0,684^{* *} \\
(-0,80-0,52)\end{array}$ & $\begin{array}{c}0,621^{* *} \\
(0,43-0,76)\end{array}$ & $\begin{array}{c}0,277^{*} \\
(0,02-0,50)\end{array}$ & $\begin{array}{c}0,621^{* *} \\
(0,43-0,76)\end{array}$ \\
\hline SH & - & - & $\begin{array}{c}-0,601^{* *} \\
(-0,74-0,41)\end{array}$ & $\begin{array}{c}0,503^{* *} \\
(0,28-0,67)\end{array}$ & $\begin{array}{c}0,264 \quad(0,01- \\
0,49)\end{array}$ & $\begin{array}{c}0,568^{* *} \\
(0,37-0,72)\end{array}$ \\
\hline AG & - & - & - & $\begin{array}{c}0,544^{* *} \\
(0,33-0,70)\end{array}$ & $\begin{array}{c}0,400^{* *} \\
(0,16-0,60)\end{array}$ & $\begin{array}{c}0,773^{* *} \\
(0,64-0,86)\end{array}$ \\
\hline V5 & - & - & - & - & $\begin{array}{c}0,354^{* *} \\
(0,10-0,55)\end{array}$ & $\begin{array}{c}0,678^{* *} \\
(0,51-0,80)\end{array}$ \\
\hline V10 & - & - & - & - & - & $\begin{array}{c}0,536^{* *} \\
(0,32-0,70)\end{array}$ \\
\hline
\end{tabular}

$\mathrm{Na}$ TABELA 4 estão os resultados da regressão múltipla, apenas dos testes que foram capazes de predizer significativamente a agilidade e a velocidade de 5, 10 e $25 \mathrm{~m}$. Dois modelos foram capazes de predizer significativamente a agilidade. $\mathrm{O}$ modelo 1 , no qual foi incluído apenas o salto sêxtuplo, foi capaz de explicar $47 \%$ da variância total da agilidade ( $\mathrm{p}<0,001)$. No modelo 2 foi incluído o salto vertical $(p=0,02)$ juntamente com o salto sêxtuplo $(\mathrm{p}=0,001)$, aumentando para $50 \%$ a explicação do desempenho da agilidade.

$\mathrm{O}$ único teste capaz de predizer o desempenho da velocidade de $5 \mathrm{~m}$ foi o salto sêxtuplo ( $\mathrm{p}<$ 0,001 ), explicando $40 \%$ da variância total desta variável. Com relação à velocidade de $10 \mathrm{~m}$, apenas o salto vertical apresentou capacidade de predição significativa ( $\mathrm{p}=0,001)$, explicando $30 \%$ do desempenho. Dois modelos foram capazes de predizer o desempenho da velocidade de $25 \mathrm{~m}$. No modelo 1 foi incluído apenas o salto vertical $(\mathrm{p}=0,001)$, sendo capaz de explicar $43 \%$ do desempenho da velocidade de $25 \mathrm{~m}$. Ao acrescentar o salto sêxtuplo $(p=0,014)$ juntamente com o salto vertical ( $\mathrm{p}=0,002)$ no modelo 2 , a capacidade de explicar a velocidade de $25 \mathrm{~m}$ foi de $48 \%$. Com relação ao teste de salto horizontal, este foi o único teste que não conseguiu predizer de forma significativa $(\mathrm{p}>0,05)$ nenhuma das variáveis dependentes, tanto para a agilidade quanto para a velocidade.

Os valores $\beta$ da regressão Múltipla (TABELA 4) indicaram que o aumento de $1 \mathrm{~cm}$ do salto sêxtuplo aumenta em 0,005 s (modelo 1) ou 0,003 s (modelo 2) e aumento de $1 \mathrm{~cm}$ no salto vertical aumenta em 0,045 s (modelo 2) o desempenho da agilidade. $\mathrm{O}$ aumento de $1 \mathrm{~cm}$ no desempenho do salto sêxtuplo resulta em aumento de $0,001 \mathrm{~m} / \mathrm{s}$ na velocidade de 5 m e o aumento de $1 \mathrm{~cm}$ no salto vertical aumenta em $0,035 \mathrm{~m} / \mathrm{s}$ a velocidade de $10 \mathrm{~m}$. Por fim, o aumento de $1 \mathrm{~cm}$ no salto vertical aumenta o desempenho da velocidade de $25 \mathrm{~m}$ em 0,050 m/s (modelo 1 ), enquanto que no modelo 2 , o aumento de $1 \mathrm{~cm}$ do salto vertical resulta em aumento de $0,030 \mathrm{~m} / \mathrm{s}$ e para o salto sêxtuplo $0,001 \mathrm{~m} / \mathrm{s}$. 
TABELA 4 - Resultados da Regressão Múltipla tendo como variáveis independentes (VI) o salto vertical (SV), salto horizontal (SH), salto sêxtuplo (SS), e como variáveis dependentes (VD) a agilidade (AG) e a velocidade de 5 (V5), 10 (V10) e $25 \mathrm{~m}$ (V25).

Os resultados do Salto Horizontal não foram descritos uma vez que não apresentaram capacidade de predição significativa para nenhuma das variáveis dependentes. Erro Padrão referente à Constante.

\begin{tabular}{|c|c|c|c|c|c|c|c|c|}
\hline VD & Modelo & VI & Constante & $\beta$ & $\beta$ Padronizado & Erro Padrão & $\mathbf{R}^{2}$ Ajustado & $p$ \\
\hline \multirow{3}{*}{ AG } & Modelo 1 & SS & 15,690 & 0,005 & 0,684 & 0,628 & 0,467 & $<0,001$ \\
\hline & \multirow{2}{*}{ Modelo 2} & SS & \multirow{2}{*}{15,589} & 0,003 & 0,475 & \multirow{2}{*}{0,602} & \multirow{2}{*}{0,502} & $=0,001$ \\
\hline & & SV & & 0,045 & 0,312 & & & $=0,02$ \\
\hline V5 & Modelo 1 & SS & 1,949 & 0,001 & 0,642 & 0,870 & 0,401 & $<0,001$ \\
\hline V10 & Modelo 1 & SV & 3,432 & 0,035 & 0,561 & 0,267 & 0,301 & $=0,001$ \\
\hline \multirow{3}{*}{ V25 } & Modelo 1 & SV & 6,643 & 0,050 & 0,661 & 0,211 & 0,427 & $<0,001$ \\
\hline & Modelo 2 & SV & \multirow{2}{*}{7,270} & 0,030 & 0,432 & \multirow{2}{*}{0,326} & \multirow{2}{*}{0,482} & $=0,002$ \\
\hline & & SS & & 0,001 & 0,341 & & & $=0,014$ \\
\hline
\end{tabular}

\section{Discussão}

O presente estudo teve como objetivos verificar a relação dos saltos vertical, horizontal e sêxtuplo, com a agilidade e velocidade de 5, 10 e $25 \mathrm{~m}$. Além disso, verificar a capacidade dos testes de salto vertical, horizontal e sêxtuplo em predizer o desempenho da agilidade e velocidade de 5, 10 e $25 \mathrm{~m}$, em crianças. Os principais resultados indicam que o salto vertical e o salto sêxtuplo apresentaram maior relação com a agilidade e a velocidade de 5,10 e $25 \mathrm{~m}$, quando comparados com o salto horizontal. Além disso, para a agilidade e a velocidade de $5 \mathrm{~m}$, o salto sêxtuplo apresentou maior correlação. Para a velocidade de 10 e 25 $\mathrm{m}$, o salto vertical foi o teste que apresentou maiores valores. Com relação à predição do desempenho, tanto o salto vertical quanto o salto sêxtuplo foram capazes de predizer significativamente o desempenho da agilidade e da velocidade de $25 \mathrm{~m}$. Além disso, se mostraram capazes de predizer significativamente a velocidade de 5 e $10 \mathrm{~m}$, respectivamente.

Apesar dos resultados deste estudo apresentarem correlação moderada entre SV, SS, SH e agilidade, a literatura apresenta resultados contraditórios, dependendo do teste e da população estudada. Os resultados referentes à correlação dos testes $\mathrm{SV}, \mathrm{SH}$ e $S S$ com a AG $(r=-0,60$ a $-0,68)$ apresentaram valores semelhantes $(r=-0,58)$ ao do salto vertical de mulheres atletas $^{14}$, de jogadoras de futebol $r=0,61^{11}$ e atletas profissionais de basquetebol $\mathrm{r}=0,59^{18}$. No entanto, outros estudos em crianças ${ }^{22}$, jogadores de futebol ${ }^{15}$, adolescentes atletas ${ }^{17}$ e mulheres atletas de diversas modalidades ${ }^{11}$ apresentaram valores inferiores de correlação $(r=-0,36$ a 0.52) entre a agilidade e os saltos vertical e horizontal.
Da mesma forma que a agilidade, os resultados entre a velocidade e o salto vertical apresentam resultados contraditórios na literatura. Alguns estudos apresentaram resultados similares aos deste estudo para a correlação entre o salto vertical com os testes de velocidade de $10 \mathrm{~m}(\mathrm{r}=0,44$ vs $\mathrm{r}=0,47)$ em jogadores de futebol ${ }^{23}, 25 \mathrm{~m}(\mathrm{r}=0,64 \mathrm{vs} \mathrm{r}=-0,66)$ em mulheres jogadoras de futebol ${ }^{11}$. Apesar de no do presente estudo a maior distância analisada ser de $25 \mathrm{~m}$, esta apresentou valores similares comparado a $30 \mathrm{~m}$, sendo de $\mathrm{r}=0,64$ vs $\mathrm{r}=-0,62^{18,23}, \mathrm{r}=0,64$ vs $0,60^{24}$ e para a distância de $35 \mathrm{~m}, \mathrm{r}=0,64$ vs $-0,62^{25}$ em jogadores de futebol.

Os resultados contrários aos do presente estudo $(r=0,43)$ para a correlação entre o salto vertical e velocidade de $10 \mathrm{~m}$ são de $\mathrm{r}=-0,13^{26}, \mathrm{r}=0,29^{27}, \mathrm{r}$ $=-0,62^{16}$ e $r=0,72^{24}$. Na comparação entre a velocidade de $5 \mathrm{~m}$ e o salto vertical a correlação verificada no presente estudo $(r=0,51)$ foi inferior a obtida por CRONIN e HANSEN ${ }^{16}, r=-0,60$, enquanto que na comparação com a velocidade de $30 \mathrm{~m}$ os valores de correlação foram de $r=0,44^{27}$ e $r=0,56^{16}$, valores inferiores aos verificados neste estudo para a velocidade de $25 \mathrm{~m}(\mathrm{r}=0,64)$. Na comparação entre o salto horizontal e a velocidade de $25 \mathrm{~m}$, os resultados do presente estudo $(r=0,57)$ e os da literatura, $r=$ $-0,30^{15}$ e $\mathrm{r}=0,700^{17}$ também diferem ente si.

A divergência entre os resultados deste e de outros estudos, no que se refere à variabilidade da relação entre diferentes tipos de saltos com a agilidade e velocidade demonstra a complexidade em predizer este tipo de desempenho, o qual é determinado por diferentes fatores. Os fatores associados ao desempenho da 
agilidade e velocidade são a transferência rápida de energia ${ }^{28}$, predominância de fibra muscular de contração rápida, aumento da excitabilidade do motoneurônio e velocidade de condução nervosa ${ }^{29}$, desenvolvimento de força concêntrica ${ }^{30}$, potência e torque muscular ${ }^{31}$, utilização do ciclo alongamento encurtamento ${ }^{32}$, tempo de contato com o solo ${ }^{33}$ e condição de treinamento ${ }^{34}$.

Dentre os fatores determinantes do desempenho, um dos aspectos que é comum ao desempenho do salto, da velocidade e da agilidade é a utilização da energia elástica, produzida pelo ciclo de alongamento e encurtamento para produzir força ${ }^{32}$. Este fato poderia explicar a capacidade dos testes de salto vertical e salto sêxtuplo em predizer, parcialmente, o desempenho da agilidade e da velocidade, sendo de 30 a $50 \%$. De fato, a energia elástica é uma característica comum do desempenho dos saltos, da agilidade e da velocidade. No entanto, não deve ser analisada como único mecanismo responsável pelas relaçōes existentes. A utilização da energia elástica também esta presente na execução do salto horizontal, o qual não apresentou capacidade de predição da agilidade e velocidade. Outra característica dos saltos que deve ser considerada é o tempo necessário para realizar o salto medido por meio do tempo de contato com o solo, que é $30 \%$ menor para o salto vertical, comparado ao salto horizontal ${ }^{35}$. Isto possivelmente pode explicar a capacidade do teste de salto vertical em predizer a agilidade e velocidade, capacidades as quais requerem pouco tempo de contato com o solo durante a execução dos movimentos ${ }^{33,36}$. No entanto, não há informações sobre o tempo de contato com o solo durante o teste de salto sêxtuplo.

As características determinantes do desempenho dos saltos, agilidade e velocidade, tais como os fatores neurais, estão presentes, principalmente, em atletas treinados para executar "sprints", o que não ocorre em indivíduos não treinados ${ }^{29}$. Este fato pode explicar a discrepância nos resultados encontrados por diferentes estudos que analisaram a relação entre saltos, agilidade e velocidade, uma vez que foram estudadas amostras com atletas de diferentes modalidades e com idades distintas, além de amostras não participantes de treinamento físico sistematizado, como a utilizada no presente estudo.

Com relação à predição do desempenho, os resultados desse estudo demonstraram que tanto o SV quanto o SS foram capazes de predizer significativamente o desempenho da AG. Este resultado corrobora com os resultados obtidos por BARNES et al. ${ }^{14}$, no qual o teste de salto vertical foi o único capaz de predizer a agilidade, em comparação ao salto em profundidade de $30 \mathrm{~cm}$ e pico de força isométrica de extensão das pernas, em jogadores de voleibol. Quanto ao teste $\mathrm{SH}$, ele não foi eficiente em predizer a AG, resultado similar ao verificado por MARKOVIC et al. ${ }^{8}$, em homens adultos. No entanto, Ré et al. ${ }^{17}$, verificaram que o teste de salto horizontal é um bom preditor da agilidade em crianças e adolescentes. Apesar disso, no estudo realizado por Ré et al. ${ }^{17}$ apenas o teste de salto horizontal foi utilizado para predizer o desempenho da agilidade, ao contrário do presente estudo, em que foram utilizados três tipos de saltos e os resultados indicaram que o salto horizontal não foi capaz de predizer nenhuma das variáveis analisadas. $\mathrm{O}$ modelo de estudo que utiliza apenas uma modalidade de salto, não permite identificar qual salto melhor prediz o desempenho da agilidade, o que limita suas conclusões.

$\mathrm{Na}$ análise da predição da velocidade, o teste SS foi capaz de predizer a velocidade de 5 e $25 \mathrm{~m}$ e o $S V$ foi capaz de predizer significativamente a velocidade de 10 e $25 \mathrm{~m}$. Tais resultados são semelhantes aos obtidos por Palazzi e Mendez-Villanueva ${ }^{37}$ em atletas de futebol, os quais indicaram que o teste de salto vertical foi o melhor preditor nos testes de 5, 15 e 20 $\mathrm{m}$, quando comparado ao torque máximo e potência máxima isocinética de $60^{\circ}$ e $300^{\circ}$. Da mesma forma, a potência média obtida por meio do salto vertical contra movimento também é eficiente em predizer a velocidade de $10 \mathrm{~m}$, em atletas da prova de $100 \mathrm{~m}^{26}$. Por outro lado, o teste SH também não foi capaz de predizer o desempenho no teste de velocidade, ao contrário dos resultados obtidos por Ré et al. ${ }^{17}$.

Apesar do teste de salto horizontal não ter sido capaz de predizer os resultados dos testes de velocidade e agilidade, ele tem sido recomendado para estimar a potência de membros inferiores em crianças, por ser um gesto motor menos complexo comparado ao Salto Vertical ${ }^{1}$. De fato, no presente estudo o teste de salto horizontal apresentou correlação forte e significativa com o salto vertical e salto sêxtuplo, com valores de $\mathrm{r}$ $=0,73$ e $\mathrm{r}=0,77$, respectivamente, indicando que o teste é um indicador da potência de membros inferiores em crianças. Tais resultados corroboram com os de CASTRO-PiñERO et al. ${ }^{38}$, os quais indicaram que o salto horizontal é fortemente correlacionado com os testes de salto vertical, "Squat" e contra movimento $(r=0,83$ a 0,86). Desta forma, o teste de salto horizontal pode ser utilizado na avaliação e controle do treinamento, com o objetivo de avaliar a potência dos membros inferiores. No entanto, de acordo com nossos resultados, não é um teste específico para a avaliação de crianças envolvidas em treinamento de modalidades esportivas 
em que as capacidades de agilidade e velocidade de 5 , 10 e 25 m são determinantes.

De modo contrário ao salto horizontal e, apesar da semelhança entre os movimentos dos saltos horizontal e sêxtuplo, o salto sêxtuplo foi capaz de predizer a agilidade e velocidade de 5 e $25 \mathrm{~m}$. A literatura sobre o teste de salto sêxtuplo diz respeito apenas a sensibilidade desse teste ao treinamento da modalidade esportiva Handebol ${ }^{39}$. Este foi o primeiro estudo a demonstrar a eficiência do teste de salto sêxtuplo em predizer o desempenho da agilidade e velocidade de 5 e $25 \mathrm{~m}$, uma vez que não foram encontradas na literatura informações sobre a capacidade do teste de salto sêxtuplo em predizer o desempenho da agilidade e velocidade.

Além de serem utilizados como forma de avaliação e controle do treinamento, saltos são utilizados como exercícios durante as sessóes de treinamento, por serem eficazes em aumentar a potência de membros inferiores e a velocidade de diferentes distâncias ${ }^{40-41}$. O desempenho da velocidade e da agilidade apresenta correlação com a capacidade de força ${ }^{12}$. No presente estudo, de acordo com os valores de $\beta$ apresentados na TABELA 4, o aumento de $1 \mathrm{~cm}$ no desempenho do Salto Vertical resulta em aumento de $0,045 \mathrm{~s}$ no desempenho da agilidade, $0,035 \mathrm{~m} / \mathrm{s}$ na velocidade de $10 \mathrm{~m}$ e $0,050 \mathrm{~m} / \mathrm{s}$ na velocidade de $25 \mathrm{~m}$. Já o aumento de $1 \mathrm{~cm}$ no salto sêxtuplo aumenta em 0,005 $s$ a agilidade, $0,001 \mathrm{~m} / \mathrm{s}$ a velocidade de $5 \mathrm{~m}$ e 0,001 $\mathrm{m} / \mathrm{s}$ a velocidade de $25 \mathrm{~m}$, na amostra estudada. Estes são indicativos de que a utilização de saltos como meio de treinamento em crianças e o consequente aumento da altura dos saltos vertical e sêxtuplo pode melhorar o desempenho da agilidade e velocidade, como já descrito recentemente em crianças $^{20} \mathrm{e}$ atletas velocistas ${ }^{19}$.

Os resultados do presente estudo sugerem que o salto sêxtuplo e o salto vertical apresentam capacidade de predição da velocidade de 5 e $10 \mathrm{~m}$ respectivamente, e ambos predizem de forma significativa a agilidade e a velocidade de $25 \mathrm{~m}$. Desta forma, tais testes podem ser utilizados para avaliação e controle do treinamento de crianças envolvidas em treinamentos de atividades que demandam agilidade e velocidade, devido à especificidade apresentada.

Devido à característica da idade da amostra, a falta da avaliação do estágio maturacional dos participantes pode ser apontada como uma limitação do presente estudo, devido à influência que a maturação sexual exerce sobre o desempenho motor ${ }^{42}$. Apesar de ainda não ter sido descrito se a relação entre o desempenho dos saltos, a velocidade e a agilidade se mantém linear em amostras de ambos os sexos com diferentes estágios maturacionais, este pode ser um fator que interfira na análise de amostras heterogêneas. No entanto, de acordo com a idade média da amostra estudada (9,5 anos), possivelmente, os participantes ainda não alcançaram a maturação sexual, uma vez que a mudança dos estágios maturacionais inicia-se aos 11 $\operatorname{anos}^{43}$. Da mesma forma, a característica homogênea das variáveis analisadas nos meninos e nas meninas, é outro indicativo deles estarem na fase pré púberes, uma vez que, com o avançar do estágio maturacional surgem diferenças significativas na força muscular ${ }^{44}$.

Além da maturação sexual, a não inclusão de um índice fisiológico ou biomecânico ${ }^{29,33}$ nos modelos de predição da agilidade e velocidade de 5, 10 e 25 $\mathrm{m}$ podem ser vistos com uma limitação do presente estudo. Possivelmente, tais índices aumentariam o poder de predição dos modelos estudados, que foram de 30 a 50\%, e tornariam possível a comparação da capacidade predição do desempenho da agilidade e velocidade entre testes de campo e laboratoriais ${ }^{37}$. Mesmo assim, a capacidade apresentada pelos testes analisados em predizer a agilidade e a velocidade é relevante, devido à aplicação prática dos testes de salto vertical, horizontal e sêxtuplo. Novos estudos, com objetivos de verificar a capacidade dos testes com diferentes tipos de saltos em predizer o desempenho da agilidade, velocidade de diferentes distâncias e outras tarefas motoras com crianças e adolescentes, em diferentes níveis de treinamento são necessários, uma vez que a maioria dos estudos na literatura foi realizada com adultos.

Os testes de salto vertical e sêxtuplo apresentaram eficácia em predizer a velocidade de 5 e $10 \mathrm{~m}$, respectivamente. Da mesma forma, ambos foram capazes de predizer a agilidade e velocidade de 25 $\mathrm{m}$, enquanto que o teste de salto horizontal não foi capaz de predizer nenhuma das variáveis estudadas. Os resultados desse estudo indicam que os testes de salto vertical e salto sêxtuplo podem ser utilizados para avaliação e controle do treinamento em crianças que praticam atividades que demandam agilidade e velocidade. Além disso, sugere-se que o treinamento de força com objetivo de aumentar o salto vertical e sêxtuplo pode contribuir para aumento do desempenho da agilidade e velocidade em crianças. 


\section{Abstract}

Relationship of vertical, horizontal and sextuple jumps with agility and speed in children

The aim of the present study were: 1) To verify the relationship of vertical, horizontal and sextuple jumps with agility and velocity of 5,10 and $25 \mathrm{~m} ; 2$ ) To verify the capacity of these jumps to predict the agility and 5,10 and $25 \mathrm{~m}$ velocity performance in children. Twenty eight boys ( $9.47 \pm 0.64$ years) and thirty girls ( $9.69 \pm 0.70$ years) were evaluated. The correlation values between agility and velocity on 5,10 and $25 \mathrm{~m}$ velocity were, respectively, $r=0.63,0.51,0.44$ and 0.64 with vertical jump, $r=$ $0.68,0.62,0.28$ and 0.62 with sextuple jump, and $r=0.60,0.50,0.26$ and 0.57 with horizontal jump. The vertical and sextuple jumps were able to predict the agility and $25 \mathrm{~m}$ velocity performance $(\mathrm{p}<$ $0.05)$. Furthermore, they demonstrated capacity to predict 5 and $10 \mathrm{~m}$ velocity, respectively $(p<0.05)$. The vertical and sextuple jump tests may be used for assessment and control of training with children practicing activities that require agility and velocity, since both jumps predicted the agility and velocity performance, which did not occur with the horizontal jump.

KEY WORDS: Running; Motor performance; Lower limbs power.

\section{Referências}

1. Guedes DP. Implicações associadas ao acompanhamento do desempenho motor de crianças e adolescentes. Rev Bras Educ Fís Esporte. 2007;21:37-60.

2. Komi PV. Força e potência no esporte. 2a ed. Porto Alegre: Artmed; 2006.

3. Zatsiorsky VM. Biomecânica da força e do treinamento de força. In: Komi PV. Força e potência no esporte. 2a ed. Porto Alegre: Artmed; 2006. Cap. 23, p. 455-502.

4. Knuttgen HG, Komi PV. Considerações básicas sobre o exercício. In: Komi PV. Força e potência no esporte. 2a ed. Porto Alegre: Artmed; 2006. Cap. 1, p. 17-21.

5. Komi PV. Stretch-shortening cycle: a powerful model to study normal and fatigued muscle. J Biomech. 2000;33:1197-206.

6. Holm I, Fredriksen P, Fosdahl M, Vøllestad N. A normative sample of isotonic and isokinetic muscle strength measurements in children 7 to 12 years of age. Acta Paediatr. 2008;97:602-7.

7. Guila J, Marques A, Prista A. Efeitos de um programa de treino de força em contexto escolar. Um estudo em crianças e adolescentes dos 12 aos 14 anos da cidade de Maputo, Moçambique. Rev Port Ciênc Desporto. 2004;4(supl.):193.

8. Markovic G, Jukic I, Milanovic D, Metikos D. Effects of sprint and plyometric training on muscle function and athletic performance. J Strength Cond Res. 2007;21:543-9.

9. Santos EJ, Janeira MA. Effects of complex training on explosive strength in adolescent male basketball players. J Strength Cond Res. 2008;22:903-9.

10. Oxyzoglou N, Kanioglou K, Ore G. Velocity, agility and flexibility performance after handball training versus physical education program for preadolescent children. Percept Mot Skills. 2009;108:873-7.

11. Vescovi JD, McGuigan MR. Relationships between sprinting, agility, and jump ability in female athletes. J Sports Sci. 2008;26:97-107.

12. Peterson MD, Alvar BA, Rhea MR. The contribution of maximal force production to explosive movement among young collegiate athletes. J Strength Cond Res. 2006;20:867-73.

13. Sheppard JM, Young WB. Agility literature review: classifications, training and testing. J Sports Sci. 2006;24:919-32.

14. Barnes JL, Schilling BK, Falvo MJ, Weiss LW, Creasy AK, Fry AC. Relationship of jumping and agility performance in female volleyball athletes. J Strength Cond Res. 2007;21:1192-6.

15. Marques MC, Travassos B, Almeida R. A força explosiva, velocidade e capacidades motoras específicas em futebolistas juniores amadores: Um estudo correlacional. Motricidade. 2010;6:5-12.

16. Cronin JB, Hansen KT. Strength and power predictors of sports speed. J Strength Cond Res. 2005;19:349-57.

17. Ré AHN, Bojikian LP, Teixeira CP, Böhme MTS. Relações entre crescimento, desempenho motor, maturação biológica e idade cronológica em jovens do sexo masculino. Rev Bras Educ Fís Esporte. 2005;19:153-62. 
18. Alemdaroğlu U. The relationship between muscle strength, anaerobic performance, agility, sprint ability and vertical jump performance in professional basketball players. J Hum Kinet. 2012;31:149-58.

19. Markström JL, Olsson CJ. Counter movement jump peak force relative to body weight and jump height as predictors for sprint running performances. (In)homogeneity of track and field athletes? J Strength Cond Res. 2012; Jun 11, Epub 2012 Jun 11. PubMed PMID: 22692108.

20. Johnson BA, Salzberg CL, Stevenson DA. A systematic review: plyometric training programs for young children. J Strength Cond Res. 2011;25:2623-33.

21. Tritschler K. Medida e avaliação em educação física e esportes de Barrow \& McGee. 5a ed. São Paulo: Manole; 2003.

22. Hazar F. The relationship of jumping and agility in children. Sci Mov Health. 2009;9:138-41.

23. Silva-Junior CJ, Palma A, Costa P, et al. Relação entre as potências de sprint e salto vertical em jovens atletas de futebol. Motricidade. 2011;7:5-13.

24. Wisløff U, Castagna C, Helgerud J, Jones R, Hoff J. Strong correlation of maximal squat strength with sprint performance and vertical jump height in elite soccer players. Br J Sports Med. 2004;38:285-8.

25. Pupo JD, Almeida CMP, Detanico D, Silva JF, Guglielmo LGA, Santos SG. Potência muscular e capacidade de sprints repetidos em jogadores de futebol. Rev Bras Cineantropom Desempenho Hum. 2010;12:255-61.

26. Maulder PS, Bradshaw EJ, Keogh J. Jump kinetic determinants of sprint acceleration performance from starting blocks in male sprinters. J Sports Sci Med. 2006;5:359-66.

27. Coelho DB, Coelho LGM, Braga ML, et al. Correlação entre o desempenho de jogadores de futebol no teste de sprint de 30m e no teste de salto vertical. Motriz. 2011:17:63-70.

28. Crowther GJ, Jubrias SA, Gronka RK, Conley KE. A “functional biopsy” of muscle properties in sprinters and distance runners. Med Sci Sports Exerc. 2002;34:1719-24.

29. Ross A, Leveritt M, Riek S. Neural influences on sprint running: training adaptations and acute responses. Sports Med. 2001;31:409-25.

30. Sleivert G, Taingahue M. The relationship between maximal jump-squat power and sprint acceleration in athletes. Eur J Appl Physiol. 2004;91:46-52.

31. De Villarreal ES, Kellis E, Kraemer WJ, Izquierdo M. Determining variables of plyometric training for improving vertical jump height performance: a meta-analysis. J Strength Cond Res. 2009;23:495-506.

32. Wilson JM, Flanagan EP. The role of elastic energy in activities with high force and power requirements: a brief review. J Strength Cond Res. 2008;22:1705-15.

33. Lockie RG, Murphy AJ, Knight TJ, De Jonge XA. Factors that differentiate acceleration ability in field sport athletes. J Strength Cond Res. 2011;25:2704-14.

34. Lehance C, Binet J, Bury T, Croisier JL. Muscular strength, functional performances and injury risk in professional and junior elite soccer players. Scand J Med Sci Sports. 2009;19:243-51.

35. Nagano A, Komura T, Fukashiro S. Optimal coordination of maximal-effort horizontal and vertical jump motions: a computer simulation study. Biomed Eng Online. 2007;6:1-9.

36. Rimmer E, Sleivert G. Effects of a Plyometrics Intervention Program on Sprint Performance. J Strength Cond Res. 2000;14:295-301.

37. Palazzi DA, Mendez-Villanueva JA. Muscle mechanical determinants of sprint performance across the professional life-span in soccer players. Doha: Qatar Foundation Annual Research Forum Proceedings; 2011. p. BMP40.

38. Castro-Piñero J, Ortega FB, Artero EG, et al. Assessing muscular strength in youth: usefulness of standing long jump as a general index of muscular fitness. J Strength Cond Res. 2010;24:810-7.

39. Souza J, Gomes AC, Leme L, Silva SG. Alterações em variáveis motoras e metabólicas induzidas pelo treinamento durante um macrociclo em jogadores de handebol. Rev Bras Med Esporte. 2006;12:129-34.

40. Chelly MS, Ghenem MA, Abid K, Hermassi S, Tabka Z, Shephard RJ. Effects of in-season short-term plyometric training program on leg power, jump- and sprint performance of soccer players. J Strength Cond Res. 2010;24:2670-6.

41. Sedano S, Matheu A, Redondo JC, Cuadrado G. Effects of plyometric training on explosive strength, acceleration capacity and kicking speed in young elite soccer players. J Sports Med Phys Fitness. 2011;51:50-8.

42. Jones MA, Hitchen PJ, Stratton G. The importance of considering biological maturity when assessing physical fitness measures in girls and boys aged 10 to 16 years. Ann Hum Biol. 2000;27:57-65.

43. Pinto ICS, Arruda IKG, Diniz AS, Cavalcanti AMTS. Prevalência de excesso de peso e obesidade abdominal, segundo parâmetros antropométricos, e associação com maturação sexual em adolescentes escolares. Cad Saúde Pública. 2010;26:1727-37.

44. Temfemo A, Hugues J, Chardon K, Mandengue SH, Ahmaidi S. Relationship between vertical jumping performance and anthropometric characteristics during growth in boys and girls. Eur J Pediatr. 2009;168:457-464. 
Relação dos saltos vertical, horizontal e sêxtuplo com a agilidade

ENDEREÇO

Diogo Henrique Constantino Coledam R. Delaine Negro, 90 apto. 123B 86055-680 - Londrina - PR - BRASIL e-mail: diogohcc@yahoo.com.br
Recebido para publicação: 16/03/2012

Revisão: 01/07/2012

Aceito: 10/08/2012 